\title{
Entretien avec Pierre Léna
}

Josette Le Coq et Daniel Malbert

\section{OpenEdition}

Journals

Édition électronique

URL : http://journals.openedition.org/ries/3338

DOI : 10.4000/ries.3338

ISSN : 2261-4265

\section{Éditeur}

Centre international d'études pédagogiques

\section{Édition imprimée}

Date de publication : 1 juin 1997

Pagination : 31-40

ISSN : 1254-4590

\section{Référence électronique}

Josette Le Coq et Daniel Malbert, « Entretien avec Pierre Léna », Revue internationale d'éducation de Sèvres [En ligne], 14 | 1997, mis en ligne le 30 juillet 2013, consulté le 03 mai 2019. URL : http:// journals.openedition.org/ries/3338; DOI : 10.4000/ries.3338

Ce document a été généré automatiquement le 3 mai 2019.

(c) Tous droits réservés 


\title{
Entretien avec Pierre Léna
}

\author{
Josette Le Coq et Daniel Malbert
}

$1 \quad$ L'intérêt du public pour la science vous semble-t-il plus fort aujourd'hui?

C'est vraiment le jour où poser la question après l'annonce du clonage de la brebis Dolly. Oui, l'intérêt est indiscutable, avec un côté un peu Janus de séduction/crainte. Que ce soient les avancées scientifiques ou techniques proprement dites avec les possibilités qu'elles offrent - fascinantes et parfois effrayantes -, que ce soient les conséquences sociales du développement technique, de l'automation sur l'emploi, ou que ce soit l'aspect proprement intellectuel de découverte du nouveau (en biologie, en astrophysique par exemple), on peut dire que l'intérêt du public est considérable. On en a des signes qui ne trompent pas: le succès des revues de vulgarisation scientifique, des livres et même des émissions à la télévision depuis trois ou quatre ans.

$2 \quad$ N'y a-t-il pas aussi un danger dans cette médiatisation?

Il y a beaucoup de choses à dire à ce sujet. Le problème de la science - et de la technique d'ailleurs -, c'est qu'elle est compliquée. Je dis souvent que les mots de la science sont des mots qui ont un sens précis, souvent dérivé du vocabulaire courant, mais avec un contenu assez différent. Prenez le mot «information» par exemple: le concept scientifique d'information a quelque chose à voir avec l'emploi courant du terme, mais il est quand même beaucoup plus précis, plus particulier et ne se prête pas à n'importe quel traitement. Et c'est le cas de beaucoup d'autres termes de la science. La science parle de bien des objets que l'on ne voit jamais, de plus en plus éloignés de l'expérience sensible. Demandez à vos amis quels sont les mots de la science qu'ils évoquent spontanément, vous entendrez : gènes, virus, quasars, trous noirs, circuits intégrés. Ce sont des mots qui traînent dans le quotidien mais ce sont des mots de l'invisible, du microscopique ou de l'infiniment grand, des mots très abstraits. Et on peut dire la même chose des objets de la technique : qui aujourd'hui peut accéder à ce que contient n'importe lequel de ces objets que l'on manipule tous les jours, une calculette, une télécommande de télévision, un ordinateur bien évidemment, mais aussi le programmateur du four micro-ondes, les micro-ondes elles-mêmes? Il y a quantité d'objets techniques qui sont de jolies boîtes avec quelques boutons et des écrans, mais sur le contenu desquels on ne sait plus rien. 
3 Il y a donc une opacité du monde scientifique et technique, des mots de la science et des objets de la technique qui est assez redoutable. C'est pourquoi je crois que toute cette démarche de vulgarisation, de "mise en culture de la science", selon l'expression de Jean-Marc Lévy-Leblond, est extrêmement importante pour que nos concitoyens ne soient pas dans un monde totalement étranger. Parce que l'étrangeté autorise n'importe quel discours, y compris le plus délirant. Ce n'est pas seulement vrai de la science. Sur ce qui est étrange ou étranger, on finit par dire n'importe quoi, par construire des logiques soit de menace, d'inquiétude, soit de fausse appropriation. C'est sans doute ce qui fait le succès des "fausses sciences ", qui sont en fait un simulacre d'appropriation d'un monde devenu trop opaque pour être pénétrable.

Les scientifiques savent-ils, aujourd'hui, parler de la science?

Cette démarche de vulgarisation doit respecter une certaine déontologie, adopter un souci de rigueur. Le niveau moyen de ce qui se fait en France n'est pas trop mauvais de ce point de vue. Le monde scientifique lui-même a certainement beaucoup évolué sur la nécessité d'une plus grande transparence, d'une meilleure présentation de son activité au public. Il y a vingt-cinq ans, pour un chercheur au CNRS, aller vers les médias ou se consacrer à la vulgarisation était perçu de façon plutôt négative par l'institution. Dans le pire des cas, comme une prostitution indue de son savoir et, au mieux, comme du temps perdu ou aimablement dépensé à des choses secondaires. Cela a beaucoup changé, avec des aspects un peu pervers: comme partout aujourd'hui, l'image des choses finit par avoir plus de valeur que les choses elles-mêmes. Les institutions se sont dotées de communications scientifiques « externes » et il devient parfois plus important d'avoir un nouveau résultat publié à la première page de France Soir ou du Monde que dans une revue scientifique. Le système a bien intégré l'idée que - par pur souci de survie d'ailleurs - la science ne peut pas vivre dans sa bulle si confortable, si fascinante, en ignorant que cette bulle est quand même plongée dans une société dont elle vit, qu'elle transforme profondément et à laquelle elle doit des comptes. Le monde scientifique doit veiller à ce que l'opacité ne mène pas à l'étrangeté et finalement au rejet.

5 La science devrait-elle être "portée » par la société ? Pensez-vous que le néophyte a une chance face à la science?

J'aime y croire... En fait, la société subit la science. L’appétence pour la connaissance, le nouveau, les découvertes est de tous temps, mais l'impact de la science sur la vie quotidienne de chacun est vraiment nouveau et il est subi. Parce que les scientifiques courent très loin devant tout le monde. Une conviction personnelle : je crois que si la bulle de la science continue à se développer en laissant tout le monde derrière, le processus s'arrêtera. Il y a d'ailleurs des tas de petits signes que les scientifiques sentent bien : s'ils ne s'expliquent pas, la société finira par les considérer comme dangereux ou inutiles et diminuera leurs crédits.

6 La vraie question, c'est de savoir si on peut encore transmettre une science qui est devenue si complexe. Je crois qu'il faut essayer, qu'il faut qu'on invente des formes de médiation. La Fête de la science a été une tentative en ce sens en France. L'école est un autre lieu où organiser cette médiation.

$7 \quad$ L'interdisciplinarité à l'école va-t-elle dans ce sens?

Il faut distinguer les différents niveaux d'enseignement. Nous parlerons plus loin de l'école primaire. Dans le secondaire, on commence à reconnaître, en France, qu'enseigner les acquis scientifiques comme des vérités intemporelles - ce qu'ils sont d'ailleurs en partie - n'est probablement pas la bonne approche pédagogique et qu'il faut insérer un 
état donné de la science dans l'histoire qui y a conduit: c'est un changement assez profond par rapport à la science telle qu'elle m'a été enseignée dans les années cinquante quand j'étais au lycée, c'est en particulier une réhabilitation de l'histoire des sciences. Cette idée fait son chemin année après année mais n'a pas encore eu la traduction concrète qui est nécessaire, et les étudiants scientifiques n'ont pas tous la possibilité de suivre des cours d'histoire des sciences liés aux disciplines qu'ils étudient parce qu'il n'y a pas de chaire d'histoire des sciences dans les universités françaises. Tant que cet enseignement ne sera pas donné, les futurs professeurs n'y auront pas accès ; cela restera des tentatives individuelles sans véritable impact sur l'évolution de l'enseignement. Il y a au sein des équipes de philosophes toute une tradition de publications qui intéressent les scientifiques mais cela ne se traduit pas dans la formation des étudiants scientifiques ni dans le cursus des instituts universitaires de formation des maitres.

8 L'usage de la langue est aussi très important. Les enseignants de premier cycle universitaire scientifique constatent souvent l'incapacité majeure des étudiants à écrire en français une argumentation scientifique; peut-être sont-ils bons en français par ailleurs, mais leur pensée scientifique est construite de manière extrêmement maladroite par rapport à la langue, comme si l'acquisition de la science et l'acquisition de la langue étaient deux choses disjointes. Notre tradition d'enseignement scientifique est vraiment très formelle : c'est une tradition dans laquelle tout ce qui ne se mathématise pas n'a pas vraiment d'importance. La construction de l'argumentation avec les mots de la langue passe facilement au second plan. Un travail commun du professeur de littérature et des professeurs de sciences permettrait aux élèves de parvenir à une meilleure aisance d'expression.

9 Les mathématiques étant elles-mêmes une langue, on y fait peut-être plus attention au langage, à la rigueur de l'argumentation, au choix des mots. Les autres sciences, les sciences de la nature, utilisent les mathématiques comme un langage mais ne s'y réduisent pas et on ne peut donc pas les faire entrer entièrement dans un canon mathématique. C'est le problème d'un enseignement trop formel de la physique : on fait de la physique mathématique mais ce n'est plus de la physique. Que dire aussi de la biologie qui, elle, n'entre pas ou à peine dans la formulation mathématique et dont l'enseignement s'en trouve décalé? La formalisation, qui est nécessaire, sert souvent d'écran à la compréhension de l'argumentation.

10 N'y a-t-il pas en France une difficulté à intégrer l'expérimentation à l'enseignement?

La manipulation de l'objet est fondamentale dans l'éducation scientifique. Le rapport au réel, à la résistance du réel est central.

11 À Paris VII, il y a quelques années, nous proposions à chacun de nos étudiants de premier cycle un budget de cent francs avec lequel il devait mettre au point une expérience, faire un exposé oral qui réponde à cette nécessité d'argumenter en français ce que l'on fait, ce que l'on voit et ce que l'on en déduit, produire un texte écrit et une manipulation qui fonctionne. On a vu des choses extraordinaires: le réveil en particulier de jeunes très handicapés par rapport à ce que l'on appelle aujourd'hui l'intégration. Ils trouvaient tout à coup un rapport au réel différent de l'image qu'ils se faisaient du savoir : quelque chose de lointain, d'inaccessible. Ils développaient soudain un rapport réel avec leurs mains et leurs yeux, leurs sens. Tout cela est bien exploité dans les musées scientifiques américains tel que l'Exploratorium'.

12 Très curieusement, on a dans notre système scolaire une tradition d'enseignement assez formel des connaissances et en même temps, la France a toujours produit des 
scientifiques expérimentateurs de très grand talent: on peut citer Georges Charpak, Pierre-Gilles de Gennes mais aussi, dès le XIX siècle, Fresnel et Fizeau. Ce talent de bricoleur génial existe donc. Malgré une pratique très formelle de l'enseignement, les lycées n'ont pas complètement perdu cette tradition : quand j'étais président de la Société de physique, nous avons lancé en 1989 les Olympiades de physique (en France) récompensant les élèves de première et de terminale qui réalisent un projet expérimental: on a chaque année des résultats magnifiques qui ne viennent pas seulement des grands lycées de Paris et de Lyon, mais répartis sur toute la France et venant d'établissements très divers. Donc, des talents existent du côté de la technique avec, en parallèle, en France, une dévalorisation des filières techniques qui n'existe pas en Allemagne, par exemple. Mais il est vrai que la tradition anglo-saxonne d'enseignement est plus empirique, plus près du fait expérimental que la nôtre. Il est important de s'inspirer de ces modèles pour la formation de nos scientifiques.

13 Ne fait-on pas des progrès en pédagogie?

C'est, disons, un souci. Par exemple, l'enseignement de la physique a été réformé ces dernières années, on a introduit, en classes préparatoires scientifiques, les travaux d'initiative personnelle encadrée (TIPE) : les élèves ont à choisir un sujet accepté par le professeur et à conduire une expérience et non plus un TP programmé où on remplit les blancs de la fiche. L'initiative est du côté de l'élève dans la construction d'un projet qui doit être expérimental. C'est un signe très positif. En négatif, il y a quelques années, on avait fait l'aberration de séparer la physique et la technologie, au collège, comme si la technologie était une chose en soi, la technologie devenait alors expérimentale mais de façon caricaturale car on y faisait des expériences sans contenu conceptuel, c'est, à mon avis, un désastre. Cette espèce d'isolement de l'objet technique est bien français : d'un côté, le cerveau qui pense, qui est noble et, de l'autre, la main qui exécute, qui l'est moins - il y a alors les disciplines de la main et celles de la tête.

14 Les cultures nationales n'ont-elles pas beaucoup d'influence sur l'enseignement?

C'est vrai, mais il n'empêche que nous sommes tous, aujourd'hui, confrontés à un autre problème : celui de l'écran. A l'observatoire de Meudon, vous verrez tout le monde devant un terminal, très peu de gens travaillent avec la matière; cette évolution est récente, profonde et radicale, avec une transformation du mode de travail, du rapport aux autres, des rapports internationaux. On entre dans un autre rapport à la science dont on est loin d'avoir épuisé toutes les conséquences, rapport avec la virtualité où on fait exister sur l'écran des choses qui sont peu contraintes par ce que nous appelons la "réalité » et où on peut expérimenter de manière presque intégralement libre, y compris avec les objets mathématiques. L'écran est un outil pédagogique prodigieux : au lieu de faire l'expérience sur la table, je vais la faire sur l'écran; au lieu de construire une molécule dans le tube à essai, le chimiste va la construire sur écran, il va rêver toutes les molécules possibles et, éventuellement, il finira par en réaliser ou en faire réaliser une dans le laboratoire. Comme la puissance des machines augmente de manière constante, on ne voit pas actuellement de limite et notre rapport aux choses est en train de changer. De manière ultime, les choses sont toujours là mais, vous le voyez bien à propos par exemple de clonage, la virtualité l'emporte : à partir du moment où on en connaît les mécanismes, toutes les possibilités sont explorables. Éventuellement, on réalisera une des possibilités, si on le peut ou si on le veut ou si on a le droit de le faire, mais tout le raisonnement se passe dans le monde virtuel. 

l'enseignement. En France, nous avons une grande catastrophe derrière nous : le «plan informatique pour tous à l'école » qui a rendu tous les enseignants extraordinairement allergiques à l'informatique pédagogique. Les enseignants ont des ordinateurs chez eux, les élèves aussi, mais la gestion de l'informatique dans le cadre scolaire est très confuse. On est devant une technique hyperpuissante et envahissante, qui se fait sa place sans même jouer des coudes. Comment maintenir l'équilibre ? Je ne sais pas.

C'est un problème qui se pose à tous les niveaux pédagogiques. En formation doctorale, par exemple, les étudiants préfèrent faire des simulations merveilleuses sur l'écran plutôt que d'aller observer avec un télescope. Je ne sais pas comment cela va évoluer, c'est une vraie question pour la pédagogie et elle se pose de la même manière partout, me semblet-il.

L'ordinateur ne risque-t-il pas de recréer une scission entre les gens qui ont accès à la science et ceux qui n'y ont pas accès?

L'ordinateur n'est qu'une opacité parmi d'autres dans l'infusion de la science au sein d'une culture. Je ne crois pas que ce soit pire mais, par contre, Internet contient un nouveau grand risque de désappropriation. Il y a une formidable richesse sur Internet mais c'est comme la visite du Louvre. Lâchez-y quelqu'un qui ne connaît rien à la peinture, si vous ne lui donnez pas de guide, pas d'explication, il va très vite s'ennuyeron pourrait dire cela aussi de la visite d'une cathédrale. Mais si la même visite est faite avec quelqu'un qui peut se faire guide, qui va interpréter, qui va préparer la marche d'accès à la compréhension de tel ou tel tableau, de tel ou tel chapiteau, tel ou tel mode architectural, alors tout s'ouvre, tout devient intelligible et à la mesure de la compétence du guide. De même, outre le fait qu'il est en anglais - ce qui est déjà une difficulté Internet est complètement opaque si l'on y navigue sans cadre pour structurer la connaissance acquise et donc se l'approprier. Je crois qu'il y a un risque réel : Internet est un outil extraordinaire mais il peut conduire à une coupure terrifiante entre ceux qui peuvent y pénétrer, s'en enrichir et les autres.

Il faut vraiment aider les enfants, dès leur plus jeune âge, à s'orienter dans la science. Est-ce le sens de « La main à la pâte»?

Le succès rencontré par le livre ${ }^{2}$, et pas seulement auprès des instituteurs, montre qu'il y a là un intérêt qui dépasse largement l'école. Si la démarche de réconciliation ou d'intégration de la science expérimentale que nous proposons a un écho aussi profond dans la société, cela signifie qu'elle répond à une demande. Dans beaucoup d'écoles, nous avons d'ailleurs insisté sur la nécessité d'associer les parents à l'expérience.

Il y a plusieurs raisons à cela : dans l'enseignement des sciences expérimentales tel qu'on essaie de le promouvoir (sans changer les programmes, qui sont très convenables) la démarche de l'enseignant est vraiment très nouvelle, puisqu'on lui propose d'accepter de dire «je ne sais pas» devant la question de l'enfant et de dire «nous allons chercher ensemble». Beaucoup d'instituteurs que j'ai rencontrés sont insécurisés par cette situation. Leur propre formation scientifique est souvent lointaine ou inexistante, en tout cas marquée par les mêmes difficultés que j'évoquais tout à l'heure, d'opacité de mots, des concepts et des objets. Donc ils sont mal à l'aise. C'est pourquoi on a voulu associer les parents, comme d'ailleurs cela se fait beaucoup aux États-Unis, parce que on ne veut pas que les parents disent: "Eh bien, les instituteurs ne savent rien, ils ne savent pas répondre ». Il faut que les parents aussi comprennent que c'est une démarche de découverte, d'investigation (inquiry, comme disent les Américains) dans laquelle on prend 
le risque de ne pas avoir la réponse, car c'est la démarche qui est intéressante, c'est la façon de poser les questions. Et si, de temps en temps, on n'a pas la réponse ce n'est pas dramatique, on a quand même fait du chemin.

À Vaux-en-Velin et à Mantes-la-Ville, où les écoles ont beaucoup associé les parents, ceux-ci répondent, un peu étonnés, mais ils viennent! À Lyon, on a vu des parents, notamment d'enfants immigrés, que l'on n'avait jamais rencontrés à l'école. Ils sont tous venus pour parler de la science à l'école. Les parents dans cette situation ne se sentent pas confrontés à une culture lointaine. Quand on développe cette attitude expérimentale chez l'enfant, la première chose qu'il a envie de faire, c'est de recommencer à la maison. Évidemment, c'est important d'associer les familles car il y a aussi des risques. Ce n'est qu'un aspect de l'expérimentation.

21 Vous voyez que l'on touche là profondément au rapport entre la science et la société. Ce n'est pas du tout notre but d'améliorer la production de scientifiques par l'école française. Ce sera peut-être une conséquence; mais une conséquence beaucoup plus importante serait que cela transforme la pédagogie vers une pédagogie plus active: ce n'est pas nouveau, l'école nouvelle a fait cela en son temps, la pédagogie Freinet aussi, on a connu la leçon de choses... Mais la société change et la façon dont ces demandes pédagogiques se réinscrivent évolue. Il n'y a pas d'idées nouvelles dans tout cela, pas de révolution.

Dans l'école primaire, on a ciblé l'effort sur des techniques (lire, écrire, compter) en oubliant un peu ce qu'on lit et ce qu'on écrit : or on lit pour lire et on écrit pour écrire... quelque chose qui a du sens et de l'intérêt.

Dans les instructions de la direction des Écoles, l'élève doit tenir un cahier d'expériences dans lequel il note ce qu'il voit. Une question importante se pose : l'enseignant doit-il corriger les fautes d'orthographe? Et si l'enfant écrit ce qu'il croit avoir vu et que ce n'est pas ce qu'il « faut voir », que fait-on? Barre-t-on? Lui dit-on non? Il emporte ce cahier à la maison, il le montre aux parents, doit-on y laisser des choses fausses et qu'est-ce qui est faux ? Parce que, après tout, l'enseignant est dans une situation d'incertitude vis-à-vis de la science, alors s'il doit corriger, selon quel critère corrige-t-il ? C'est toute une série de questions qui se posent dont on voit bien qu'elles insécurisent les enseignants, qu'elles modifient assez profondément la situation de celui qui sait face à celui qui ne sait pas, pour, peut-être, la transformer en une démarche d'accompagnement de la curiosité. Il y des limites à cela, l'éveil est allé si loin dans ce sens que le spontanéisme pédagogique du « laisser faire » a été un aspect négatif de ce genre d'actions, mais c'est très intéressant de voir la façon dont ces questions se posent.

Quel est le sens de la présence de personnalités scientifiques de renommée dans cette entreprise?

Le monde scientifique s'est intéressé à la communication avec la société depuis dix ou quinze ans, mais, paradoxalement, il s'est moins intéressé au système éducatif parce qu'au fond il y est, d'une certaine façon, chez lui. Les disciplines en tant que disciplines constituées qui fabriquent du savoir sont assez peu soucieuses de l'enseignement secondaire et encore moins de l'enseignement primaire. Par exemple, toute la mise en place et le fonctionnement des IUFM reçoit du monde scientifique une attention à peu près nulle, ce que je trouve personnellement assez triste. J'espère œuvrer pour que cela change. Il y a une espèce d'indifférence que je comprends assez mal d'ailleurs mais elle ressemble beaucoup à celle d'il y a vingt ans vis-à-vis des médias, on peut donc espérer que cela va changer. Peu de personnes se préoccupent de l'école primaire car on se dit que ce sont des connaissances tellement élémentaires que, finalement, il n'y a pas de 
problème. Le monde scientifique s'est très peu intéressé à l'enseignement primaire et c'est un grand changement par rapport à ce qui s'est passé au XIX ${ }^{\mathrm{e}}$ siècle, notamment dans le monde anglo-saxon où, par exemple, Faraday, qui était ouvrier d'origine, a pesé de tout son poids pour que l'on prenne en charge une vision moderne de la science à l'école. Puis, on a assisté à des phénomènes analogues en France, après Jules Ferry. Depuis ces années-là, le fossé s'est creusé entre le monde scientifique et les étudiants de licence qui deviennent des professeurs d'école avec des conséquences d'autant plus graves que, aujourd'hui, $85 \%$ d'une promotion de jeunes professeurs des écoles ont une formation disciplinaire de littérature, psychologie ou sciences humaines et ce qu'ils ont connu des sciences au lycée est souvent plutôt dissuasif dans leur esprit.

On arrive à ce paradoxe que, dans une société fortement scientifique et technique, l'empreinte pédagogique initiale, entre 6 et 12 ans, des enfants qui vont vivre demain dans ce monde-là, qui vont le faire, devoir s'y préparer, en comprendre le langage, être confrontés à cette opacité dont on parlait, sont formés par un système dont les programmes contiennent des sciences et dont les enseignants, en immense majorité, sont démunis, insécurisés ou au mieux limités à un enseignement du polycopié ou de la fiche à trous; c'est quand même un paradoxe incroyable par rapport aux ambitions de l'école primaire que l'on avait énoncées il y a un siècle. Ce dysfonctionnement a motivé des académiciens à dire que cela ne peut plus durer et qu'il faut faire quelque chose, le but encore une fois n'étant pas de former, d'abord, des scientifiques pour améliorer le recrutement des filières scientifiques. Je suis tout à fait frappé par l'intérêt recueilli à l'Académie des sciences pour ces questions, par la perception de la nécessité d'agir.

Et en même temps, on ne sait pas très bien comment avancer, on énonce quelques suggestions. Le ministère voudrait une extension de l'expérience dès la rentrée prochaine. Les échos venus du terrain sont enthousiastes mais aussi conseillent la prudence. Une extension massive rapide serait, à mon sens, une catastrophe. Pourtant l'ouverture à tous est bien l'enjeu de la démocratisation de l'école. Une société ne peut pas être à ce point conditionné dans l'emploi, dans la santé, dans les rapports de la personne à l'information, conditionnée par les outils techniques et les concepts scientifiques qui les ont fondés et être, par ailleurs, faite d'analphabètes scientifiques. Parce que finalement cela mène à l'irrationnel, au discours sectaire : on n'est plus dans le domaine de l'argumentation. La science est une école rude, longue, humble mais c'est une école de justice. On ne peut pas prétendre que la pierre qui tombe ne tombe pas, on est obligé de se rallier à une évidence objective indépendante de la personne et de ses opinions. Il y a une espèce de contrainte du réel. Au travers de l'apprentissage à l'école des apports de la science, se construit la démocratie. Monge, déjà, ne s'y était pas trompé : ses textes sur la place des sciences dans l'éducation sont tout à fait consonants aujourd'hui, avec des besoins qui n'ont pas vraiment changé.

La science serait-elle un moyen privilégié d'apprentissage de la construction de la vérité?

La vérité n'est ni donnée a priori ni entièrement subjective ni entièrement intangible, elle est un peu tout cela à la fois, elle existe et pourtant elle évolue. Il faut apprendre cela aux enseignants. Naturellement, cela concerne les philosophes, naturellement, les professeurs de philosophie du secondaire ont quelque chose à dire là-dessus avec les scientifiques et les historiens. De tous temps, les philosophes se sont intéressés à la science. L'intérêt des professeurs du secondaire pour la philosophie est moindre : on retrouve ici le résultat de l'enseignement qu'ils ont suivi à l'université : enseignement de disciplines complètement cloisonnées. Le fait qu'il n'y ait pas de trace, même mineure, d'enseignement relevant des 
«sciences humaines» dans les études scientifiques est regrettable; et réciproquement d'ailleurs, le fait qu'un étudiant de lettres ou de philosophie ait pu suivre son cursus sans jamais avoir été exposé de manière un peu construite aux grandes idées sur l'organisation de la matière et du cosmos, est choquant (vingt heures de cours au premier cycle universitaire seraient raisonnables).

Vous avez parlé des réactions des parents et des enseignants. Qu'en est-il des enfants?

Je n'ai pas parlé de l'intérêt des enfants pour la science car c'est l'évidence même. La capacité d'interrogation, d'émerveillement d'enfants entre 6 et 12 ans pour tout ce qui touche à la nature est prodigieuse et cela quelle que soit leur origine culturelle. J'ai passé une matinée à Mantes-la-Ville devant quatre-vingts enfants : deux petits gaulois et toutes les couleurs de la planète ; c'était fascinant de curiosité, d'interrogations, d'interpellation avec des mots maladroits souvent glanés à la télévision. Dans leur tête ce n'était pas très clair mais nous avons parlé. C'est pour cela que l'idée de la science comme facteur d'intégration, là où beaucoup d'autres ont échoué, est à creuser. C'est une des composantes très fortes des expériences américaines que nous sommes allés voir à Chicago et Los Angeles. Cela fonctionne dans des quartiers où plus rien ne paraît possible. Mais le rapport au monde scientifique est difficile pour certaines cultures: une des grandes difficultés, et c'est pour cela qu'il faut associer les parents, est le choc culturel entre la nouveauté à l'école et la culture traditionnelle à la maison. La science est juste à la frontière de ce choc. Donc on pourrait dire à la fois que l'on a affaire, d'une part, à des intelligences complètement neuves, ambitieuses de comprendre et, d'autre part, à des cultures très peu scientifiques, souvent moins marquées par la construction de la rationalité qui baigne la nôtre depuis longtemps, et qui pourraient avoir, et ont parfois, une attitude de rejet.

La science n'est-elle pas un lieu d'objectivité, de débat où l'affectivité a une importance moindre que dans d'autres domaines?

Vous avez raison mais la science est une certaine représentation du monde et il faut entrer dans cet espace de représentation. Prenez par exemple la notion de causalité - à une cause un effet - : nous sommes cartésiens " de père en fils ", mais ce n'est pas quelque chose de spontané, nos représentations des objets, du mouvement, de l'espace, de la vitesse sont inscrites dans notre culture mais pas forcément dans toute culture. Cela peut présenter une difficulté mais, de fait, cette difficulté est moindre que dans d'autres domaines : par exemple, à Chicago, les enfants ont moins de mal à formuler ce qu'ils voient lors d'une expérience qu'à raconter leurs vacances, à supposer qu'ils en prennent. Je trouve cette piste intéressante, ce n'est pas avec cela que l'on résoudra le problème de l'intégration en France, mais c'est une petite pierre que l'on peut poser.

\section{NOTES}

1. Musée créé en 1969 à San Francisco, par Franck Oppenheimer. Voir pour plus de détails, l'article de Bernard Schiele, Les musées scientifiques, p. 105. 
2. La main à la pâte. Les sciences à l'école primaire, présenté par Georges Charpak, Paris, Flammarion, 1996.

\section{RÉSUMÉS}

Le rapport entre la science et la société est, en France, complexe : à l'opacité langagière et technique du monde scientifique s'oppose un intérêt du public qui se manifeste à travers le succès actuel de la médiatisation des sciences. Il est urgent et indispensable d'inventer, dès l'école, des «formes de médiation » entre ces deux pôles : ce peut être, notamment, réalisé par un développement de l'expérimentation scientifique dans l'enseignement.

\section{INDEX}

Index géographique : France

Mots-clés : éducation scientifique, expérimentation, pédagogie expérimentale, sciences, société vulgarisation 